PROCEEDINGS OF THE

AMERICAN MATHEMATICAL SOCIETY

Volume 126, Number 1, January 1998, Pages 305-310

S 0002-9939(98)04001-5

\title{
DISTINGUISHING EMBEDDED CURVES IN RATIONAL COMPLEX SURFACES
}

\author{
TERRY FULLER
}

(Communicated by Ronald A. Fintushel )

\begin{abstract}
We construct many pairs of smoothly embedded complex curves with the same genus and self-intersection number in the rational complex surfaces $\mathbb{C} P^{2} \# n \overline{\mathbb{C} P}^{2}$ with the property that no self-diffeomorphism of $\mathbb{C} P^{2} \# n \overline{\mathbb{C} P}^{2}$ sends one to the other. In particular, as a special case we answer a question originally posed by R. Gompf (1995) concerning genus two curves of selfintersection number 0 in $\mathbb{C} P^{2} \# 13 \overline{\mathbb{C} P}^{2}$.
\end{abstract}

\section{INTRODUCTION}

Among the many successes arising from the recent introduction of SeibergWitten invariants into 4-manifold topology has been an increased understanding of the relationship between complex surfaces and the smooth structures of their underlying 4-manifolds. In particular, if $k_{S} \in H^{2}(S, \mathbb{Z})$ denotes the canonical class of a minimal complex surface $S$ of general type, then we must have $f^{*}\left(k_{S}\right)= \pm k_{S}$ for any self-diffeomorphism $f([\mathrm{~W}])$. Similar restrictions are known to hold for most elliptic surfaces. However, for a rational complex surface $S$ constructed by blowing up $\mathbb{C} P^{2}$, it is easy to construct self-diffeomorphisms which do not preserve $k_{S}$, and it is natural to ask what restrictions exist for self-diffeomorphisms of $\mathrm{S}$ in this case. In this note, we make explicit some restrictions by describing embeddings in rational complex surfaces $\mathbb{C} P^{2} \# n \overline{\mathbb{C} P}^{2}$ of curves $C$ and $D$ of self-intersection number 0 and the same genus with the property that no self-diffeomorphism of $\mathbb{C} P^{2} \# n \overline{\mathbb{C P}}^{2}$ maps $C$ to $D$.

\section{BRANCHED COVERS}

We begin by constructing a family of complex surfaces. For all $k \geq 0$, we denote the $k$ th Hirzebruch surface, namely the holomorphic $S^{2}$-bundle over $S^{2}$ with a section of self-intersection number $-k$, by $\mathbb{F}_{k}$. If $F$ is a fiber of $\mathbb{F}_{k}$, and $\Delta_{k}$ is the section with $\Delta_{k}^{2}=k$, then $[F]$ and $\left[\Delta_{k}\right]$ form a basis for $H_{2}\left(\mathbb{F}_{k}, \mathbb{Z}\right)$ with $[F]^{2}=0$ and $[F] \cdot\left[\Delta_{k}\right]=1$.

A curve representing the homology class $a[F]+b\left[\Delta_{k}\right] \in H_{2}\left(\mathbb{F}_{k}, \mathbb{Z}\right)$ is referred to as a curve of type $(a, b)$, or simply as an $(a, b)$ curve. We can construct a smooth

Received by the editors April 22, 1996 and, in revised form, July 9, 1996.

1991 Mathematics Subject Classification. Primary 57R40; Secondary 14J26.

Key words and phrases. Rational complex surface, embedded surface, branched cover, normal sum.

(C) 1998 American Mathematical Society 
$(a, b)$ curve by beginning with $a$ copies of $F$ and $b$ copies of $\Delta_{k}$ in general position. This is represented by $a+b$ spheres which meet in

$$
a b+k(1+2+\cdots+(b-1))=a b+\frac{k}{2}(b-1) b
$$

transverse positive double points. Resolving each of these nodes by trading the intersection point for a handle (this standard procedure may be done holomorphically $[\mathrm{KM}]$ ) produces a connected smoothly embedded curve of genus

$$
(a-1)(b-1)+\frac{k}{2}(b-1) b .
$$

We define $X_{k}(2 p, 2 q)$ to be the 2 -fold cover of $\mathbb{F}_{k}$, branched over this construction of a curve of type $(2 p, 2 q)$. For all $k \geq 0$, and $p, q \geq 2, X_{k}(2 p, 2 q)$ is a simply connected minimal complex surface $[\mathrm{P}]$.

\section{BRANCHED COVERS AS NORMAL SUMS}

We begin with some lemmas.

Lemma 1. Let $D_{1}$ and $D_{2}$ be two smoothly embedded disks in $B^{4}$ with $\partial D_{1} \cup \partial D_{2} \subset$ $S^{3}$, and assume that $D_{1}$ and $D_{2}$ intersect once in a positive transverse double point p. Let $D$ denote the annulus obtained by resolving that double point, and let $\widetilde{D}$ denote the proper transform of $D_{1} \cup D_{2}$ when $B^{4}$ is blown up at $p$. Then the 2fold cover of $B^{4}$ branched over $D$ is diffeomorphic to the 2-fold cover of $B^{4} \# \overline{\mathbb{C} P}^{2}$ branched over $\widetilde{D}$.

Proof. By the construction of $D$, it is isotopic to the annulus in $S^{3}$ pictured in Figure 1(a). Hence by the techniques in [AK], the 2-fold cover of $B^{4}$ branched over $D$ is diffeomorphic to the handlebody in Figure 1(b), the $D^{2}$-bundle over $S^{2}$ with Euler number -2 .

Figure 2(a) shows $\widetilde{D}$ in $B^{4} \# \overline{\mathbb{C P}}^{2}$, drawn as two disjoint disks. Since the branch set $\widetilde{D}$ is disconnected, the double branched cover in Figure 2(b) requires a 1-handle. Cancelling that 1-handle against one of the 2-handles completes the proof.

We also need a lemma from algebraic geometry.

Lemma 2. Two curves in $\mathbb{F}_{k} \# n \overline{\mathbb{C P}}^{2}$ are linearly equivalent if and only if they are homologous. In particular, if $C_{1}$ and $C_{2}$ are homologous smooth curves of selfintersection number 0 , then $C_{1}$ is parallel to $C_{2}$.

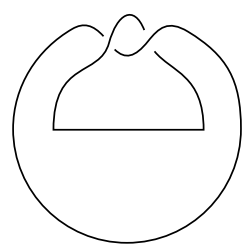

(a)

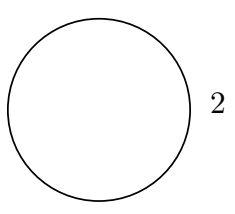

(b)

FIGURE 1 


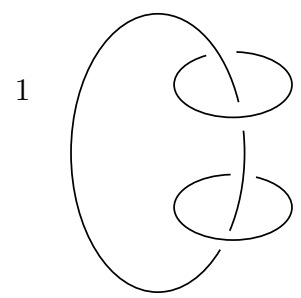

(a)

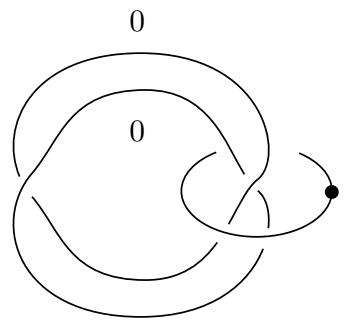

(b)

FiguRe 2

Proof. The first statement is a standard application of the long exact cohomology sequence associated to the short exact exponential sheaf sequence

$$
0 \longrightarrow \mathbb{Z} \longrightarrow \mathcal{O} \stackrel{\exp }{\longrightarrow} \mathcal{O}^{*} \longrightarrow 0,
$$

where $\mathcal{O}$ is the sheaf of holomorphic functions on $\mathbb{F}_{k} \# n \overline{\mathbb{C P}}^{2}$, and $\mathcal{O}^{*}$ is the sheaf of nonzero holomorphic functions on $\mathbb{F}_{k} \# n \overline{\mathbb{C P}}^{2}$ ([GH], p. 518). For the second statement, since $C_{1}$ and $C_{2}$ are linearly equivalent, $C_{1}$ and $C_{2}$ are both members of a pencil $\left\{D_{\alpha}\right\}_{\alpha \in \mathbb{C} P^{1}}$. Since $C_{i}^{2}=0$, this pencil is in fact a singular fibration over $\mathbb{C} P^{1}$ with (by Bertini's Theorem) only finitely many singular fibers. Hence, since $C_{1}$ and $C_{2}$ are nonsingular fibers, this fibration determines a trivialization of the normal bundle of $C_{1}$, with $C_{2}$ a parallel copy of $C_{1}$.

We next recall a well known cut-and-paste procedure. Let $M_{1}$ and $M_{2}$ be smooth 4-manifolds and let $N_{1}$ and $N_{2}$ be smoothly embedded genus $g$ surfaces in $M_{1}$ and $M_{2}$, respectively, satisfying $N_{1}^{2}=-N_{2}^{2}$. Then there is an orientation-reversing isomorphism of regular neighborhoods

$$
\psi: \nu\left(N_{1}\right) \rightarrow \nu\left(N_{2}\right) .
$$

We then form the normal sum of $M_{1}$ and $M_{2}$ along $N_{1}$ and $N_{2}$ by gluing $M_{1}-\stackrel{\circ}{\nu}\left(N_{1}\right)$ to $M_{2}-\stackrel{\circ}{\nu}\left(N_{2}\right)$ using

$$
\left.\psi\right|_{\partial \nu\left(N_{1}\right)}: \partial \nu\left(N_{1}\right) \rightarrow \partial \nu\left(N_{2}\right) .
$$

In general, the diffeomorphism type of the resulting manifold depends on $\psi$ and is denoted $M_{1} \#_{\psi} M_{2}$. By [G], this operation may be performed in the symplectic category. In our applications, we have $M_{1}=M_{2}=M$, and $N_{1}=N_{2}=N$ with $N^{2}=0$, so $\nu(N) \cong \Sigma \times D^{2}$, where $\Sigma$ is a genus $g$ surface. We can therefore form the normal sum of two copies of $\mathrm{M}$ along $\mathrm{N}$ using

$$
i d_{\Sigma} \times \sigma: \Sigma \times S^{1} \rightarrow \Sigma \times S^{1},
$$

where $\sigma$ is complex conjugation. In this case we write $M \#_{N} M$ for the normal sum. In the case where $N$ is a nonsingular fiber in a singular genus $g$ fibration of $M$, this operation is known as a fiber sum.

Lemma 3. Let $M$ be a smooth 4-manifold, and $N$ a smoothly embedded genus $g$ surface in $M$ with $N^{2}=0$. Let $N^{\prime}$ denote a parallel copy of $N$. Then $M \#_{N} M$ is diffeomorphic to the 2-fold cover of $M$ branched over $N \cup N^{\prime}$. 
Proof. There is an obvious free involution on two copies of $M-\stackrel{\circ}{\nu}(N)$, which becomes an involution with fixed point set $\Sigma \times\{ \pm 1\}$ when the identifications used to form the normal sum are made. Furthermore, the quotient of the normal sum under this involution is diffeomorphic to $M$. Hence the normal sum $M \#_{N} M$ is diffeomorphic to the double cover of $M$ branched over $N \cup N^{\prime}$.

Let $P_{n}$ denote the rational surface $\mathbb{C} P^{2} \# n \overline{\mathbb{C} P}^{2}$.

Proposition 4. $X_{k}(2 p, 2 q)$ is diffeomorphic to the normal sum of two copies of the rational surface $P_{k q^{2}+2 p q+1}$ along a smooth connected curve $C$, where $C$ has self-intersection number 0 , and

$$
\operatorname{genus}(C)=(p-1)(q-1)+\frac{k}{2}(q-1) q .
$$

Proof. By construction, $X_{k}(2 p, 2 q)$ is the double cover of $\mathbb{F}_{k}$, branched over a canonical smoothing of a $(2 p, 2 q)$ curve, as described before. By Lemma 1, we may instead blow up some of these double points prior to taking the double branched cover. To do this carefully, we again begin by taking $2 p$ copies of $F$ and $2 q$ copies of $\Delta_{k}$ in general position. This represents a curve of type $(2 p, 2 q)$ by $2 p+2 q$ (individually) embedded spheres which meet in $4 p q+k q(2 q-1)$ transverse positive double points. (We get $4 p q$ intersections from the intersection of $F$ and $\Delta_{k}$, and $\frac{k}{2}(2 q-1)(2 q)$ intersections from the normal bundle of $\Delta_{k}$.)

We next picture this $(2 p, 2 q)$ curve as a $(p, q)$ curve together with a push-off of itself. Doing this, we count that $2 p q+k(q-1) q$ of the double points are selfintersections of the $(p, q)$ curve or of its push-off, and $k q^{2}+2 p q$ of the double points are intersections of the $(p, q)$ curve with its push-off. If we resolve all of the former double points, and blow up all of the latter, the result is two disjoint smooth curves $B$ and $B^{\prime}$ in $\mathbb{F}_{k} \#\left(k q^{2}+2 p q\right) \overline{\mathbb{C P}}^{2}$. Note that each curve individually can be described by beginning with a $(p, q)$ curve in $\mathbb{F}_{k}$ represented by $p$ copies of $F$ and $q$ copies of $\Delta_{k}$, resolving all $p q+\frac{k}{2}(q-1) q$ of its double points, and blowing up $k q^{2}+2 p q$ smooth points. We calculate

$$
B^{2}=\left(B^{\prime}\right)^{2}=\left(p[F]+q\left[\Delta_{k}\right]\right)^{2}-\left(k q^{2}+2 p q\right)=0,
$$

and

$$
\operatorname{genus}(B)=\operatorname{genus}\left(B^{\prime}\right)=(p-1)(q-1)+\frac{k}{2}(q-1) q .
$$

So from Lemma $2, B$ and $B^{\prime}$ are parallel. Hence by Lemma 3 the normal sum of two copies of $\mathbb{F}_{k} \#\left(k q^{2}+2 p q\right) \overline{\mathbb{C} P}^{2}$ along $B$ is diffeomorphic to the double cover of $\mathbb{F}_{k} \#\left(k q^{2}+2 p q\right) \overline{\mathbb{C} P}^{2}$ branched over $B \cup B^{\prime}$, and hence to $X_{k}(2 p, 2 q)$.

We complete the proof by noting that there is a biholomorphic equivalence

$$
\mathbb{F}_{k} \#\left(k q^{2}+2 p q\right) \overline{\mathbb{C P}}^{2} \cong P_{k q^{2}+2 p q+1},
$$

and that the image of $B$ under this equivalence is a smooth curve $C$ with the same genus and self-intersection number.

\section{Distinguishing Embedded CuRves}

In this section we first describe, for comparison with $C$, an explicit construction of embedded curves $D$ in $P_{k q^{2}+2 p q+1}$. Let $g=\operatorname{genus}(C)=(p-1)(q-1)+\frac{k}{2}(q-1) q$. 
We can represent a degree $d$ curve in $\mathbb{C} P^{2}$ by an immersed sphere with $\frac{1}{2}(d-1)(d-2)$ positive double points, with $d$ chosen so that

$$
\frac{(d-1)(d-2)}{2}>g
$$

We then resolve $g$ of these nodes to obtain an immersed genus $g$ curve with

$$
\frac{(d-1)(d-2)}{2}-g
$$

positive double points. Blowing up these remaining double points produces an embedded genus $g$ curve of square

$$
d^{2}+4 g-2(d-1)(d-2) .
$$

If $d$ is also chosen so that this number is non-negative, then we let $D$ be the curve of square 0 obtained via additional blow ups. One can check that the total number of blow ups used in this construction is

$$
d^{2}-3\left(\frac{(d-1)(d-2)}{2}-g\right) .
$$

If this number is strictly less than $k q^{2}+2 p q+1$, then we view $D$ as a curve in $P_{k q^{2}+2 p q+1}$.

Theorem 5. There is no self-diffeomorphism of $P_{k q^{2}+2 p q+1}$ that sends $C$ to $D$.

Proof. Assuming there is a diffeomorphism of $P_{k q^{2}+2 p q+1}$ sending $C$ to $D$, then

$$
\left(P_{k q^{2}+2 p q+1}\right) \#_{C}\left(P_{k q^{2}+2 p q+1}\right) \cong\left(P_{k q^{2}+2 p q+1}\right) \#_{D}\left(P_{k q^{2}+2 p q+1}\right) .
$$

However, any normal sum of $P_{k q^{2}+2 p q+1}$ along $D$ is necessarily nonminimal, since by construction $D$ requires fewer than $k q^{2}+2 p q+1$ blow ups, and so we can find an exceptional curve $E$ in $P_{k q^{2}+2 p q+1}$ disjoint from $D$. Since

$$
\left(P_{k q^{2}+2 p q+1}\right) \#_{C}\left(P_{k q^{2}+2 p q+1}\right) \cong X_{k}(2 p, 2 q)
$$

is minimal, this is a contradiction.

\section{REMARKS}

1. We illustrate these constructions for curves of genus 2 in $P_{13}$. Setting $k=$ $0, p=3$, and $q=2$, we recall the construction of Proposition 4. Beginning with a $(3,2)$ curve in $\mathbb{F}_{0}=\mathbb{C} P^{1} \times \mathbb{C} P^{1}$ represented by 3 copies of $F$ and 2 copies of $\Delta_{0}$, we resolve all 6 double points, and then blow up the resulting genus 2 curve 12 times to obtain an embedded genus 2 curve $B$ of square 0 in $\left(\mathbb{C} P^{1} \times \mathbb{C} P^{1}\right) \# 12 \overline{\mathbb{C P}}^{2}$. We then let $C$ be the image of $B$ under the biholomorphic equivalence

$$
\left(\mathbb{C} P^{1} \times \mathbb{C} P^{1}\right) \# 12 \overline{\mathbb{C} P}^{2} \cong P_{13} .
$$

To construct $D$, we begin with a degree 6 curve in $\mathbb{C} P^{2}$, represented by 6 complex lines meeting in 15 transverse double points. Resolving 7 of these yields an immersed genus 2 surface with 8 nodes. Blowing up these produces an embedding of a genus 2 surface of square $36-32=4$ in $P_{8}$. Four more blow ups and we have an embedded surface $D$ in $P_{12}$ (and hence in $P_{13}$ ) of square 0 . In [G], Gompf asked if there is a symplectomorphism of $P_{13}$ (i.e. a self-diffeomorphism of $P_{13}$ preserving the usual symplectic structure) sending $C$ to $D$. Applying Theorem 5 shows that such a self-diffeomorphism is not possible. 
(The construction of $C$ in $[\mathrm{G}]$ is different from ours. Gompf constructs $C$ by blowing up a degree 4 curve represented by an immersed genus 2 surface with one node, and it can be seen with Kirby calculus that his construction and the one given here are the same. Also, as noted in $[\mathrm{G}]$, we can obtain an embedded genus 2 curve by beginning with a degree 5 curve in $\mathbb{C} P^{2}$. However, a degree 5 curve with 4 nodes is birationally equivalent to a degree 4 curve with one node, so this merely gives another construction of $C$. )

2. By setting $k=0, p=g+1$, and $q=2$, the above construction gives distinguishable curves $C$ and $D$ in $P_{4 g+5}$ for any genus $g$. To see this, it can be checked that in this case one can find an integer $d$ satisfying the various inequalities involved in the construction of $D$. We omit this unpleasant bit of algebra.

3. Proposition 4 generalizes a result of Ron Stern [S], who has obtained explicit constructions of certain elliptic surfaces and Horikawa surfaces as normal sums (in fact, as fiber sums) of rational surfaces.

\section{ACKNOWLEDGEMENT}

This paper was a chapter in the author's Ph.D. thesis at The University of Texas at Austin. The author would like to thank Ron Stern, Bob Gompf, and Yat-Sun Poon for helpful conversations.

\section{REFERENCES}

[AK] S. Akbulut and R. Kirby, Branched covers of surfaces in 4-manifolds, Math. Ann. 252 (1980), 111-131. MR 82j:57001

[G] R. Gompf, A new construction of symplectic manifolds, Ann. of Math 142 (1995), 527-595. MR 96j:57025

[GH] P. Griffiths and J. Harris, Principles of algebraic geometry, John Wiley \& Sons, Inc, New York, 1978. MR 80b:14001

[KM] P. Kronheimer and T. Mrowka, Gauge theory for embedded surfaces, II, Topology 34 (1995), 37-97. MR 96b:57038

[P] U. Persson, Chern invariants of surfaces of general type, Compos. Math. 43 (1981), 3-58. MR 83b:14012

[S] R. Stern, personal communication.

[W] E. Witten, Monopoles and four-manifolds, Math. Res. Letters 1 (1995), 769-796. MR 96d:57035

Department of Mathematics, University of Texas at Austin, Austin, Texas 78712

Current address: Department of Mathematics, University of California, Irvine, California 92717

E-mail address: tfuller@math.uci.edu 